\title{
Dram1 confers resistance to Salmonella infection
}

\author{
Samrah Masud, Rui Zhang, Tomasz K. Prajsnar, and Annemarie H. Meijer* \\ Institute of Biology Leiden, Leiden University, Einsteinweg 55, 2333 CC, Leiden, The Netherlands \\ * for correspondence: a.h.meijer@biology.leidenuniv.nl
}

\begin{abstract}
Dram1 is a stress and infection inducible autophagy modulator that functions downstream of transcription factors p53 and NFKB. Using a zebrafish embryo infection model, we have previously shown that Dram1 provides protection against the intracellular pathogen Mycobacterium marinum by promoting the p62-dependent xenophagy of bacteria that have escaped into the cytosol. However, the possible interplay between Dram1 and other antibacterial autophagic mechanisms remains unknown. Recently, LC3-associated phagocytosis (LAP) has emerged as an important host defense mechanism that requires components of the autophagy machinery and targets bacteria directly in phagosomes. Our previous work established LAP as the main autophagic mechanism by which macrophages restrict growth of Salmonella Typhimurium in a systemically infected zebrafish host. We therefore employed this infection model to investigate the possible role of Dram1 in LAP. Morpholino knockdown or CRISPR/Cas9-mediated mutation of Dram1 led to reduced host survival and increased bacterial burden during $S$. Typhimurium infections. In contrast, overexpression of dram 1 by mRNA injection curtailed Salmonella replication and reduced mortality of the infected host. During the early response to infection, GFP-Lc3 levels in transgenic zebrafish larvae correlated with the dram1 expression level, showing over two-fold reduction of GFP-Lc3-Salmonella association in dram 1 knockdown or mutant embryos and an approximately $30 \%$ increase by dram1 overexpression. Since LAP is known to require the activity of the phagosomal NADPH oxidase, we used a Salmonella biosensor strain to detect bacterial exposure to reactive oxygen species (ROS) and found that the ROS response was largely abolished in the absence of dram1. Together, these results demonstrate the host protective role of Dram1 during $S$. Typhimurium infection and suggest a functional link between Dram 1 and the induction of LAP.
\end{abstract}




\section{Introduction}

Autophagy has long been known as a fundamental housekeeping process wherein dysfunctional cellular components are captured inside double membrane vesicles that fuse with lysosomes to degrade and recycle the contents (Levine and Klionsky, 2004; Mizushima, 2007). More recently, autophagy has also become recognized as an integral part of the immune system, functioning not only as a direct anti-microbial mechanism but also contributing to regulation of the immune response (Deretic et al., 2013). Autophagy can function as a non-specific bulk process or as a selective mechanism mediated by receptors that recognize molecular degradation signals like ubiquitin (Boyle and Randow, 2013). The selective autophagy of invading microbes, referred to as xenophagy, is an innate immune effector mechanism targeting invading microbes either when present inside membranebound compartments or when they escape into the host cytosol (Huang and Brumell, 2014). A hallmark of all forms of autophagy is the conjugation of microtubule-associated protein 1 light chain 3 (MAP1LC3, hereafter LC3) with phosphatidylethanolamine on the autophagosomal double membrane structures (Mizushima et al., 2004 ). However, the autophagy machinery can also recruit LC3 to single membrane compartments, specifically phagosomes, in a process known as LC3-associated phagocytosis (LAP) (Sanjuan et al., 2007; Martinez, 2018; Upadhyay and Philips, 2019). Both xenophagy and LAP have been shown to be important for resistance to infections, but pathogens have also evolved mechanisms to subvert these host autophagic defenses (Huang and Brumell, 2014; Mostowy, 2013; Deretic et al., 2006; Hubber et al., 2017; Prajsnar et al., 2020).

There are various mechanisms by which autophagy can be induced in response to internal and external stress factors, such as nutrient restriction, DNA damage, and microbial invaders. One factor implicated in the activation of autophagy is DNA damage regulated autophagy modulator gene 1 (DRAM1), which encodes a member of an evolutionary conserved family of six transmembrane proteins (Mah et al., 2012). DRAM1 was first reported as direct target gene of the tumor suppressor protein p53 and shown to play a role in p53-mediated autophagy and apoptosis (Crighton et al., 2006). DRAM1 has been implicated in several types of cancers (Galavotti et al., 2013; Chen et al., 2018; Meng et al., 2018). Overexpression of DRAM1 has been shown to increase basal levels of autophagosome numbers, indicating that DRAM1 can act early in the autophagy process, contributing to autophagosome formation (Mah et al., 2012). However, the DRAM1 protein predominantly localizes to lysosomes and there is evidence also for functions at later steps in the process, showing that DRAM1 enhances autophagic flux and promotes ATPase activity and lysosomal acidification (Zhang et al., 2013; Geng et al., 2020). DRAM1 is thought to mediate crosstalk between autophagy and apoptosis by interacting with the proapoptotic protein BAX (Guan et al., 2015). Furthermore, in an autophagy-independent manner, DRAM1 interacts with transporter proteins to promote lysosomal amino acid efflux, which activates the nutrient-sensing complex mTORC1 (Beaumatin et al., 2019). Other molecular interactions of DRAM1 that could explain its mode of action at early as well as late steps of the autophagy pathway remain to be elucidated. 
In addition to DNA damage, DRAM1 is also induced by infection (Laforge et al., 2013; van der Vaart et al., 2014). In HIV-infected T-cells, DRAM1 has been shown to function downstream of p53, triggering lysosomal membrane permeabilization and cell death (Laforge et al., 2013). In contrast, we have shown that the induction of DRAM1 in macrophages infected with Mycobacterium tuberculosis is independent of p53 and mediated instead by transcription factor NFKB, which functions downstream of pathogen recognition by Toll-like receptor (TLR) signaling (van der Vaart et al., 2014). Similarly, induction of the zebrafish homolog of DRAM1 (dram1) by Mycobacterium marinum infection relies on the TLR adaptor molecule MyD88 (van der Vaart et al., 2014). M. marinum-infected zebrafish embryos develop a tuberculosislike disease and we have shown that Dram1 plays a host protective role in this model (van der Vaart et al., 2014; Meijer and van der Vaart, 2014; Zhang et al., 2020). Specifically, we found that knockdown of dram1 strongly reduces the co-localization of Lc3 with M. marinum, whereas overexpression increases LC3-M. marinum co-localization in a manner dependent on the selective autophagy receptor p62 (Meijer and van der Vaart, 2014). Additionally, we observed that in dram1-/- CRISPR mutant zebrafish, not only Lc3 co-localization but also the acidification of $M$. marinum-containing vesicles was reduced (Zhang et al., 2020). Failing to contain the intracellular infection, macrophages in dram1-/- zebrafish succumbed to pyroptotic cell death (Zhang et al., 2020).

In the present study we sought to investigate the role of Dram1 during infection of zebrafish embryos with another intracellular pathogen, Salmonella enterica serovar Typhimurium (S. Typhimurium). A zebrafish model for $S$. Typhimurium infection has previously been established and dram 1 expression is inducible following intravenous injection of the pathogen in this model (van der Sar et al., 2003; Stockhammer et al., 2010). In our recent work, we have shown that LAP is the main autophagic process targeting $S$. Typhimurium following phagocytosis of the pathogen by macrophages in the zebrafish host (Masud et al., 2019a). Furthermore, we found that inhibition of LAP, by knockdown of factors specific for this process (Rubicon and NADPH oxidase), impaired host resistance (Masud et al., 2019a; Masud et al., 2019b). Here, we demonstrate that Dram1 is required for host resistance to $S$. Typhimurium infection and promotes LC3 association with this pathogen, supporting a role for Dram1 in LAP-mediated host defense.

\section{Results}

Knockdown and overexpression of dram1 indicates its function in host defense against Salmonella infection

In order to investigate the role of Dram1 during Salmonella infections we modulated levels of dram1 by splice blocking morpholino knockdown or mRNA injection at the 1-2-cell stage. We challenged the host at 2 days post fertilization ( $2 \mathrm{dpf}$ ) with $S$. Typhimurium by intravenous injection and recorded progression of the resulting infection by survival curves and CFU 
determination (Figure 1A). We found that dram1 knockdown resulted in hypersusceptibility to Salmonella infection, where at 48 hours post infection (hpi) nearly $74 \%$ of hosts succumbed to $S$. Typhimurium infection in significant contrast to $54 \%$ of mortality in control hosts (Figure 1B). Moreover, only $4 \%$ of dram1-deprived hosts were alive at 72 hpi compared to $29 \%$ of the control group (Figure 1B). Additionally, under knockdown conditions of dram1, infected hosts contained significantly higher $S$. Typhimurium bacterial counts at 24 hpi (Figure 1C). Conversely, the overexpression of dram 1 by mRNA injection resulted in higher survival rates (Figure 1B) and lower numbers of $S$. Typhimurium bacteria at $24 \mathrm{hpi}$ (Figure $1 \mathrm{C}$ ). These results indicate that Dram1 restricts Salmonella infection.

\section{Dram1 is required for the LC3 response of the host that targets Salmonella phagocytosed by macrophages}

We have previously shown that the autophagy response of macrophages towards Salmonella in our model occurs mainly as Lc3-associated phagocytosis (LAP) (Masud et al., 2019a). To study whether Dram1 contributes to the LC3 targeting of Salmonella, we injected dram1 morpholino into embryos of the Tg(CMV:GFP-maplc3b1) line (hereafter referred to as GFPLC3) and infected these with $m$ Cherry-expressing $S$. Typhimurium (Figure 2A). We observed that GFP-LC3 associations with Salmonella cells were significantly Dram1 dependent, since morpholino knockdown resulted in more than two-fold reduction of GFP-Lc3-positive infected phagocytes (Figure 2B,C,E). Thus, the increased susceptibility of dram 1 knockdown embryos (Figure 1) is associated with diminished levels of Lc3-Salmonella associations. Next, we investigated if the positive effect of $\mathrm{mRNA}$-mediated dram1 overexpression on restricting infection (Figure 1) is accompanied by an increased Lc3 response towards the pathogen. Indeed, infection of control and dram 1 overexpressing GFP-LC3 embryos showed a significant increase of GFP-LC3 associations with S. Typhimurium bacterial cells in the overexpression group (Figure $\mathbf{2} \mathbf{D}$ and $\mathbf{E}$ ). These results lead us to propose that Dram1 promotes the LAP response during Salmonella infections, resulting in a more effective host defense.

\section{Dram1 mutation recapitulates the infection phenotype of dram1 morpholino-induced knockdown}

In order to verify the results obtained using morpholino-mediated knockdown, we studied $S$. Typhimurium infection in a recently established dram 1 mutant line (Zhang et al., 2020). We infected dram 1 -/- and dram1 +/+ embryos with S. Typhimurium (Figure 3A) and observed that dram1-/- hosts showed increased mortality during $S$. Typhimurium infection as compared to dram1+/+ controls (Figure 3B). Bacterial growth determination confirmed the Salmonellarestricting role of Dram1 as significantly higher bacterial counts were retrieved from dram1/- embryos at $24 \mathrm{hpi}$ as compared to dram 1+/+ embryos (Figure 3C). The dram 1 mutants were subsequently tested for Lc3 targeting of Salmonella. We observed significantly less GFP-Lc3 associations with Salmonella cells in dram1-/- embryos compared to dram1+/+ embryos (Figure 3D-F), further confirming our initial observations using morpholino knockdown. The consistent results obtained in Dram1-deficient hosts achieved by knockdown or stable 
mutation support the function of Dram1 in LAP-mediated host defense during Salmonella infections.

\section{Dram1 is required for the phagocyte ROS response associated with LAP}

LAP strictly depends on NADPH oxidase and Rubicon-mediated generation of ROS (Martinez, 2018). Therefore, to further study the link between Dram1 and the LAP response to $S$. Typhimurium, we determined the effect of Dram1 deficiency on reactive oxygen species (ROS) generation. To this end we utilized an $S$. Typhimurium ROS biosensor strain that contains a constitutively expressed $m$ Cherry reporter and a GFP reporter that is activated when bacterial cells are exposed to ROS (Burton et al., 2014) (Figure 4A). We have previously shown that the activation of this ROS biosensor is strictly dependent on the expression of Rubicon and the NADPH oxidase component Cyba, demonstrating that this biosensor is a reliable indicator of the occurrence of LAP (Masud et al., 2019a). We observed that dram1-/- hosts were unable to activate the ROS biosensor in contrast to dram1+/+ individuals (Figure 4B-D). Together, our results demonstrate that Dram1 deficiency impairs both GFP-Lc3 recruitment and ROS generation in infected phagocytes of the zebrafish host, consistent with the proposed role of Dram1 in the LAP response to Salmonella.

\section{Discussion}

In this study, we have used a zebrafish infection model to demonstrate that the autophagy modulator Dram1 promotes host resistance to systemic Salmonella infection. This work extends our previous finding that Dram1 mediates autophagic host defense in the zebrafish tuberculosis model. Importantly, our study implicates Dram1 in an infection context where LAP is the predominant autophagic host defense response.

LAP has recently been shown to function as a crucial host defense mechanism in bacterial as well as fungal infection (Martinez et al., 2015; Hubber et al., 2017; Sprenkeler et al., 2016; Masud et al., 2019a). In LAP, the autophagy marker LC3 is recruited to phagosomes by a mechanism that requires essential components of the autophagy machinery, but is independent of the ULK1 preinitiation complex (Martinez et al., 2015). DRAM1 has been shown to promote autophagy and autophagic flux, but has not previously been implicated in LAP (Crighton et al., 2006; Zhang et al., 2013). We have previously shown that macrophages in systemically infected zebrafish embryos target $S$. Typhimurium almost exclusively by LAP (Masud et al., 2019a). Lc3 recruitment to S. Typhimurium in zebrafish macrophages is dependent on two proteins that are essential for LAP: Rubicon and p22Phox, a component of the NADPH oxidase (Martinez et al., 2015). Depletion of either of these proteins abolishes GFP-Lc3 association with S. Typhimurium and prevents activation of a bacterial ROS biosensor gene (Masud et al., 2019a). Here, we show that both these responses are also inhibited by knockdown or mutation of dram1, suggesting that Dram1 is required for the targeting of $S$. 
Typhimurium by LAP. This Dram1-mediated process is host-protective, since we found that dram1-deficient zebrafish embryos are more susceptible to $S$. Typhimurium infection and that dram1 overexpression promotes host resistance.

The molecular mechanism by which Dram1 promotes autophagic processes is currently unknown. Our data show that Dram1 is required not only for Lc3 recruitment but also for the ROS response in phagosomes. This suggests that Dram 1 functions upstream of Rubicon, which has been shown to stabilize the NADPH oxidase. An attractive hypothesis is that Dram1 interacts directly with components of the Beclin1-VPS34-UVRAG-Rubicon complex to promote the association of Rubicon with phagosomes. This hypothesis is consistent with a recent study on a member of the human DRAM family, DRAM2, which has been shown to stimulate autophagy by disrupting the association of Rubicon with the Beclin1/UVRAG complex (Kim et al., 2011). The displacement of Rubicon relieves its inhibitory function from this complex and thereby promotes the activity of the class III phosphatidylinositol 3-kinase, VPS34, which is required for autophagosome formation and maturation. If Dram 1 functions by a similar mechanism in the response to Salmonella infection, disruption of the RubiconBeclin1/Uvrag interaction would liberate Rubicon to associate with the Salmonella-containing phagosomes. This hypothesis would explain how Dram1 is able to stimulate autophagy and LAP at the same time. Another attractive hypothesis is that Dram1 stimulates the maturation of autophagosomes and LAPosomes by associating with lysosomal proteins, such as LAMP1 and LAMP2, similar to DRAM2 (Kim et al., 2011). Therefore, future work should focus on the effect of Dram1 on acidification of Salmonella-containing vesicles.

In addition to promoting LAP, it is likely that the activity of Dram1 can enhance other autophagic mechanisms against intracellular Salmonella, including the selective autophagy process that is mediated by ubiquitin receptors. Selective autophagy has been shown to function as an important defense mechanism against Salmonella in epithelial cells, which this pathogen enters by active invasion (Huang et al., 2009). Selective autophagy specifically targets cytosolic bacteria or detects membrane damage on the bacterial replication niche. In zebrafish, we have shown that this response is elicited by the escape of $M$. marinum from phagosomes and in this model Dram1 depends on the ubiquitin receptor p62 for its autophagy enhancing effect (van der Vaart et al., 2014). The above proposed interaction of Dram1 with Rubicon is consistent with positive effects on selective autophagy as well as LAP. In addition, the microbicidal capacity of Lc3-marked bacterial compartments might be enhanced by Dram1 activity, regardless of whether these compartments are of autophagosomal or phagosomal origin. This could be facilitated by delivery of ubiquitinated antimicrobial peptides through fusion with autophagosomes, which are present in increased numbers when Dram1 is overexpressed (Ponpuak et al., 2010; van der Vaart et al., 2014).

Different members of the DRAM family might have partially overlapping functions in host defense. In addition to a single copy of dram1, the zebrafish has two copies of the gene for Dram2, dram2a and dram $2 b$. Based on our RNA sequencing data of leukocyte populations 
sorted from zebrafish larvae, dram1 is the most abundantly expressed gene in macrophages, while the expression level of $d r a m 2 b$ is at least 10 -fold lower and dram $2 a$ is not detectably expressed (Rougeot et al., 2019). Furthermore, we have only found expression of dram1 to be inducible by infection (Stockhammer et al., 2010; Benard et al., 2014). Our study did not provide any indication that other members of the zebrafish Dram family might be involved in LAP, since mutation of dram1 abolished ROS biosensor activation in the Salmonellacontaining phagosomes almost completely. However, the possible role of Dram $2 a / b$ in zebrafish host defense requires further investigation in the light of the recent report that Mycobacterium tuberculosis evades autophagy by inducing the expression of a microRNA (miR144*) that targets human DRAM2 (Kim et al., 2017). This study has revealed that DRAM2 enhances antimicrobial activity in human monocyte derived macrophages, similar to the function that we have shown for Dram1 in the zebrafish host during infections with $M$. marinum and S.Typhimurium (van der Vaart et al., 2014); and this study). Therefore, accumulating evidence positions the members of the DRAM family as key players in autophagic defense against intracellular pathogens and it will be of great interest to further explore how these autophagy modulators might work in concert and how they could be therapeutically targeted.

\section{Materials and methods}

\section{Zebrafish lines and maintenance}

Zebrafish were handled in compliance with local animal welfare regulations and international guidelines specified by the EU Animal Protective Directive 2010/63/EU and maintained according to standard protocols (zfin.org). All studies were performed on embryos/larvae before the free feeding stage. Fish lines used for this study are $A B / T L$ (wild type strain), transgenic lines Tg(CMV:GFP-map1/c3b) (He \& Klionsky, 2010) Tg(mpeg1::mcherry-F)umsF001 (Bernut et al., 2014) and dram1-/- and dram1+/+ in Tg(CMV:GFP-map1/c3b) and Tg(mpeg1::mcherry-F) umsF001 backgrounds (Zhang et al., 2020). Embryos from adult fish were handled and treated pre-infection and post infection as described (Masud et al., 2019a).

\section{Bacterial strains and infection experiments}

S. Typhimurium SL1344 strains were used for this study, constitutively expressing $m$ Cherry or the mCherry marker in combination with a GFP biosensor for ROS (pkatGp-gfpOVA) (Burton et al., 2014). Culturing of bacteria, preparation of infection inocula and infection delivery were performed as described before (Masud et al., 2019a). Briefly, bacteria were resuspended in PBS supplemented with $2 \%$ polyvinylpyrrolidone-40 (PVP) to obtain the low dose (200-400 CFU, for survival curves and CFU counts experiments) or high dose (2000-4000 CFU, for imaging experiments). Bacterial inoculum was injected systemically into the caudal vein of 2 dpf anaesthetized embryos. Survival of infected larvae was recorded at 24 hour intervals. 


\section{Determination of in vivo bacterial (CFU) counts}

The in vivo bacterial counts were determined as described in (Masud et al., 2019a). Briefly, homogenized infected embryos were serially diluted and plated out on solid media to enumerate bacterial colonies.

\section{Dram1 knockdown and overexpression experiments}

Dram 1 expression was altered by injecting $1 \mathrm{nl}$ of a $0.1 \mathrm{mM}$ solution of a previously described antisense morpholino M01-dram1 (AAGGCTGGAAAACAAACGTACAGTA) or by injecting at a concentration of 100 pico grams of dram 1 mRNA in $1 \mathrm{nl}$ (van der Vaart et al., 2014).

\section{Image acquisition and image analysis}

Infected embryos were fixed at $4 \mathrm{hpi}$ for image acquisitions over the yolk sac region. A $63 \mathrm{x}$ water immersion objective (NA 1.2) with a Leica TCS SPE system was used. For quantification of GFP-LC3-Salmonella associations and ROS biosensor activation, the images acquired were analyzed through Z-stacks in Leica LAS AF Lite software and bacterial clusters were observed and manually counted in the overlay channel as previously described (Masud et al., 2019a). Max projections in the overlay channels were used for representative images.

\section{Statistical analysis}

All data sets were analyzed with Prism 7 software. Survival curves were analyzed with Log rank (Mantel-Cox) test. For CFU counts, one-way ANOVA was performed on Log-transformed data. Data of GFP-LC3-positive infected phagocytes and biosensor-positive phagocytes were analyzed with unpaired parametric t-test between two groups and for multiple groups the one-way ANOVA test was performed and corrected for multiple comparisons.

\section{Acknowledgements}

We thank Dirk Bumann (University of Basel) for sharing of the Salmonella strains used in this study, and Daniel Klionsky (University of Michigan) for the zebrafish transgenic line. We are also grateful to all members of the fish facility team for zebrafish care. S.M. was supported by a fellowship from the Higher Education Commission of Pakistan and the Bahaudin Zakriya University, Multan. R.Z. was supported by a fellowship from the China Scholarship Council and T.K.P. by an individual Marie Curie fellowship (PIEF-GA-2013-625975). 


\section{References}

Beaumatin, F., O'Prey, J., Barthet, V. J. A., Zunino, B., Parvy, J. P., Bachmann, A. M., O'Prey, M., Kania, E., Gonzalez, P. S., Macintosh, R., Lao, L. Y., Nixon, C., Lopez, J., Long, J. S., Tait, S. W. G. and Ryan, K. M. (2019) 'mTORC1 Activation Requires DRAM-1 by Facilitating Lysosomal Amino Acid Efflux', Mol Cell, 76(1), pp. 163-176 e8.

Benard, E. L., Roobol, S. J., Spaink, H. P. and Meijer, A. H. (2014) 'Phagocytosis of mycobacteria by zebrafish macrophages is dependent on the scavenger receptor Marco, a key control factor of proinflammatory signalling', Dev Comp Immunol, 47(2), pp. 223-33.

Bernut, A., Herrmann, J. L., Kissa, K., Dubremetz, J. F., Gaillard, J. L., Lutfalla, G. and Kremer, L. (2014) 'Mycobacterium abscessus cording prevents phagocytosis and promotes abscess formation', Proc Natl Acad Sci U S A, 111(10), pp. E943-52.

Boyle, K. B. and Randow, F. (2013) 'The role of 'eat-me' signals and autophagy cargo receptors in innate immunity', Curr Opin Microbiol, 16(3), pp. 339-48.

Burton, N. A., Schurmann, N., Casse, O., Steeb, A. K., Claudi, B., Zankl, J., Schmidt, A. and Bumann, D. (2014) 'Disparate impact of oxidative host defenses determines the fate of Salmonella during systemic infection in mice', Cell Host Microbe, 15(1), pp. 72-83.

Chen, C., Liang, Q. Y., Chen, H. K., Wu, P. F., Feng, Z. Y., Ma, X. M., Wu, H. R. and Zhou, G. Q. (2018) 'DRAM1 regulates the migration and invasion of hepatoblastoma cells via autophagy-EMT pathway', Oncol Lett, 16(2), pp. 2427-2433.

Crighton, D., Wilkinson, S., O'Prey, J., Syed, N., Smith, P., Harrison, P. R., Gasco, M., Garrone, O., Crook, T. and Ryan, K. M. (2006) 'DRAM, a p53-induced modulator of autophagy, is critical for apoptosis', Cell, 126(1), pp. 121-34.

Deretic, V., Saitoh, T. and Akira, S. (2013) 'Autophagy in infection, inflammation and immunity', Nat Rev Immunol, 13(10), pp. 722-37.

Deretic, V., Singh, S., Master, S., Harris, J., Roberts, E., Kyei, G., Davis, A., de Haro, S., Naylor, J., Lee, H. H. and Vergne, I. (2006) 'Mycobacterium tuberculosis inhibition of phagolysosome biogenesis and autophagy as a host defence mechanism', Cell Microbiol, 8(5), pp. 719-27.

Galavotti, S., Bartesaghi, S., Faccenda, D., Shaked-Rabi, M., Sanzone, S., McEvoy, A., Dinsdale, D., Condorelli, F., Brandner, S., Campanella, M., Grose, R., Jones, C. and Salomoni, P. (2013) 'The autophagy-associated factors DRAM1 and p62 regulate cell migration and invasion in glioblastoma stem cells', Oncogene, 32(6), pp. 699-712.

Geng, J., Zhang, R., Yuan, X., Xu, H., Zhu, Z., Wang, X., Wang, Y., Xu, G., Guo, W., Wu, J. and Qin, Z. H. (2020) 'DRAM1 plays a tumor suppressor role in NSCLC cells by promoting lysosomal degradation of EGFR', Cell Death Dis, 11(9), pp. 768.

Guan, J. J., Zhang, X. D., Sun, W., Qi, L., Wu, J. C. and Qin, Z. H. (2015) 'DRAM1 regulates apoptosis through increasing protein levels and lysosomal localization of BAX', Cell Death Dis, 6, pp. e1624.

He, C. and Klionsky, D. J. (2010) 'Analyzing autophagy in zebrafish', Autophagy, 6(5), pp. 642-4.

Huang, J. and Brumell, J. H. (2014) 'Bacteria-autophagy interplay: a battle for survival', Nat Rev Microbiol, 12(2), pp. 101-14.

Huang, J., Canadien, V., Lam, G. Y., Steinberg, B. E., Dinauer, M. C., Magalhaes, M. A., Glogauer, M., Grinstein, S. and Brumell, J. H. (2009) 'Activation of antibacterial autophagy by NADPH oxidases', Proc Natl Acad Sci U S A, 106(15), pp. 6226-31.

Hubber, A., Kubori, T., Coban, C., Matsuzawa, T., Ogawa, M., Kawabata, T., Yoshimori, T. and Nagai, H. (2017) 'Bacterial secretion system skews the fate of Legionella-containing vacuoles towards LC3associated phagocytosis', Sci Rep, 7, pp. 44795.

Kim, J., Kundu, M., Viollet, B. and Guan, K. L. (2011) 'AMPK and mTOR regulate autophagy through direct phosphorylation of Ulk1', Nat Cell Biol, 13(2), pp. 132-41.

Kim, J. K., Lee, H. M., Park, K. S., Shin, D. M., Kim, T. S., Kim, Y. S., Suh, H. W., Kim, S. Y., Kim, I. S., Kim, J. M., Son, J. W., Sohn, K. M., Jung, S. S., Chung, C., Han, S. B., Yang, C. S. and Jo, E. K. (2017) 'MIR144* inhibits antimicrobial responses against Mycobacterium tuberculosis in human 
monocytes and macrophages by targeting the autophagy protein DRAM2', Autophagy, 13(2), pp. 423-441.

Laforge, M., Limou, S., Harper, F., Casartelli, N., Rodrigues, V., Silvestre, R., Haloui, H., Zagury, J. F., Senik, A. and Estaquier, J. (2013) 'DRAM triggers lysosomal membrane permeabilization and cell death in CD4(+) T cells infected with HIV', PLoS Pathog, 9(5), pp. e1003328.

Levine, B. and Klionsky, D. J. (2004) 'Development by self-digestion: molecular mechanisms and biological functions of autophagy', Dev Cell, 6(4), pp. 463-77.

Mah, L. Y., O'Prey, J., Baudot, A. D., Hoekstra, A. and Ryan, K. M. (2012) 'DRAM-1 encodes multiple isoforms that regulate autophagy', Autophagy, 8(1), pp. 18-28.

Martinez, J. (2018) 'LAP it up, fuzz ball: a short history of LC3-associated phagocytosis', Curr Opin Immunol, 55, pp. 54-61.

Martinez, J., Malireddi, R. K., Lu, Q., Cunha, L. D., Pelletier, S., Gingras, S., Orchard, R., Guan, J. L., Tan, H., Peng, J., Kanneganti, T. D., Virgin, H. W. and Green, D. R. (2015) 'Molecular characterization of LC3-associated phagocytosis reveals distinct roles for Rubicon, NOX2 and autophagy proteins', Nat Cell Biol, 17(7), pp. 893-906.

Masud, S., Prajsnar, T. K., Torraca, V., Lamers, G. E. M., Benning, M., Van Der Vaart, M. and Meijer, A. H. (2019a) 'Macrophages target Salmonella by Lc3-associated phagocytosis in a systemic infection model', Autophagy 15(5), pp. 796-812.

Masud, S., van der Burg, L., Storm, L., Prajsnar, T. K. and Meijer, A. H. (2019b) 'Rubicon-dependent Lc3 recruitment to Salmonella-containing phagosomes is a host defense mechanism triggered independently from major bacterial virulence factors', Front Cell Infect Microbiol, 9, p. 279.

Meijer, A. H. and van der Vaart, M. (2014) 'DRAM1 promotes the targeting of mycobacteria to selective autophagy', Autophagy, 10(12), pp. 2389-91.

Meng, C., Liu, Y., Shen, Y., Liu, S., Wang, Z., Ye, Q., Liu, H., Liu, X. and Jia, L. (2018) 'MicroRNA-26b suppresses autophagy in breast cancer cells by targeting DRAM1 mRNA, and is downregulated by irradiation', Oncol Lett, 15(2), pp. 1435-1440.

Mizushima, N. (2007) 'Autophagy: process and function', Genes Dev, 21(22), pp. 2861-73.

Mizushima, N., Yamamoto, A., Matsui, M., Yoshimori, T. and Ohsumi, Y. (2004) 'In vivo analysis of autophagy in response to nutrient starvation using transgenic mice expressing a fluorescent autophagosome marker', Mol Biol Cell, 15(3), pp. 1101-11.

Mostowy, S. (2013) 'Autophagy and bacterial clearance: a not so clear picture', Cell Microbiol, 15(3), pp. 395-402.

Ponpuak, M., Davis, A. S., Roberts, E. A., Delgado, M. A., Dinkins, C., Zhao, Z., Virgin, H. W. t., Kyei, G. B., Johansen, T., Vergne, I. and Deretic, V. (2010) 'Delivery of cytosolic components by autophagic adaptor protein p62 endows autophagosomes with unique antimicrobial properties', Immunity, 32(3), pp. 329-41.

Prajsnar, T. K., Serba, J. J., Dekker, B. M., Gibson, J. F., Masud, S., Fleming, A., Johnston, S. A., Renshaw, S. A. and Meijer, A. H. (2020) 'The autophagic response to Staphylococcus aureus provides an intracellular niche in neutrophils', Autophagy, pp. 1-15.

Rougeot, J., Torraca, V., Zakrzewska, A., Kanwal, Z., Jansen, H. J., Sommer, F., Spaink, H. P. and Meijer, A. H. (2019) 'RNAseq Profiling of Leukocyte Populations in Zebrafish Larvae Reveals a cxcl11 Chemokine Gene as a Marker of Macrophage Polarization during Mycobacterial Infection', Front Immunol, 10, pp. 832.

Sanjuan, M. A., Dillon, C. P., Tait, S. W., Moshiach, S., Dorsey, F., Connell, S., Komatsu, M., Tanaka, K., Cleveland, J. L., Withoff, S. and Green, D. R. (2007) 'Toll-like receptor signalling in macrophages links the autophagy pathway to phagocytosis', Nature, 450(7173), pp. 1253-7.

Sprenkeler, E. G., Gresnigt, M. S. and van de Veerdonk, F. L. (2016) 'LC3-associated phagocytosis: a crucial mechanism for antifungal host defence against Aspergillus fumigatus', Cell Microbiol, 18(9), pp. 1208-16. 
Stockhammer, O. W., Rauwerda, H., Wittink, F. R., Breit, T. M., Meijer, A. H. and Spaink, H. P. (2010) 'Transcriptome analysis of Traf6 function in the innate immune response of zebrafish embryos', Mol Immunol, 48(1-3), pp. 179-90.

Upadhyay, S. and Philips, J. A. (2019) 'LC3-associated phagocytosis: host defense and microbial response', Curr Opin Immunol, 60, pp. 81-90.

van der Sar, A. M., Musters, R. J., van Eeden, F. J., Appelmelk, B. J., Vandenbroucke-Grauls, C. M. and Bitter, W. (2003) 'Zebrafish embryos as a model host for the real time analysis of Salmonella typhimurium infections', Cell Microbiol, 5(9), pp. 601-11.

van der Vaart, M., Korbee, C. J., Lamers, G. E., Tengeler, A. C., Hosseini, R., Haks, M. C., Ottenhoff, T. H., Spaink, H. P. and Meijer, A. H. (2014) 'The DNA damage-regulated autophagy modulator DRAM1 links mycobacterial recognition via TLR-MYD88 to autophagic defense', Cell Host Microbe, 15(6), pp. 753-67.

Zhang, R., Varela, M., Forn-Cuni, G., Torraca, V., van der Vaart, M. and Meijer, A. H. (2020) 'Deficiency in the autophagy modulator Dram1 exacerbates pyroptotic cell death of Mycobacteria-infected macrophages', Cell Death Dis, 11(4), pp. 277.

Zhang, X. D., Qi, L., Wu, J. C. and Qin, Z. H. (2013) 'DRAM1 regulates autophagy flux through lysosomes', PLoS One, 8(5), pp. e63245. 


\section{Figure legends}

Figure 1: Dram1 is required for effective host defense during Salmonella infections.

A: Workflow of experiments followed in B\&C along with the time line of developing embryos. B: Survival curves of embryos with $S$. Typhimurium infection expressing different levels of $d r a m 1$. Survival curves of control embryos were compared to dram1 knockdown and overexpressing groups. One representative of three replicates is shown $(n=50)$.C: CFU counts for infected larvae groups in B. For each of the three groups five embryos/larvae per time point were used and the log transformed CFU data are shown with the geometric mea mean per time point. One representative of three replicates is shown. Error bars represent SD. $* * * P<0.001, * P<0.05$.

Figure 2: Dram1 modulates GFP-Lc3 associations with S. Typhimurium. A: Workflow and time line of experiments in B-E. B-D:. Representative confocal micrographs of $T g(C M V: G F P-L c 3 m a p 1 b)$ embryos from control (B), dram1 kd (C), and dram1 mRNA (D) groups infected with mCherryexpressing S. Typhimurium at 4 hpi. E: Quantification of GFP-Lc3-Salmonella associations at 4 hpi. Five embryos per group were imaged over the yolk sac circulation valley and quantified as percentages of infected phagocytes positive for GFP-Lc3-Salmonella associations (LC3+ve) over the total number of infected phagocytes. Error bars represent SD. One representative of three replicates is shown. Scale bar $(B-D)=10 \mu \mathrm{m} .{ }^{*} * \mathrm{P}<0.01$.

Figure 3: LAP induction in impaired in dram1 mutants. A: Workflow and time line of experiments in B-F. B: Survival curves of dram1+/+ and dram1-/- embryos infected with S. Typhimurium. One representative of two replicates is shown $(n=50)$. C: CFU counts recovered from $d r a m 1+/+$ and dram1 - /- infected individuals. Five embryos/larvae per time point were used and the log transformed CFU data are shown with the geometric mean per time point. One representative of three replicates is shown. Error bars represent SD. D,E: Representative confocal micrographs of GFP-Lc3-Salmonella associations in dram1+/+ (D) and dram1-/- (E) embryos in Tg(CMV:GFPLc3map1b background. F: Quantification of GFP-Lc3-Salmonella associations in dram1+/+ and dram1 -/- embryos. Five embryos were imaged over the yolk sac circulation valley and infected phagocytes positive for GFP-Lc3-Salmonella associations were quantified as percentage over the total number of infected phagocytes. Error bars represent SD. One representative of two replicates is shown. Scale bar $(D, E)=10 \mu \mathrm{m}, * * * * P<0.0001 . * * P<0.01, * P<0.05$

Figure 4: ROS generation is mediated by Dram1 during Salmonella infection. A: Workflow an time line of experiments in B-D. B-C:. Representative micrograph for dram1+/+ (B) and dram1-/- (C) where embryos were infected with a Salmonella biosensor strain for ROS. Inactive biosensor constitutively expresses mCherry and activated biosensor expresses GFP in addition to mCherry. Images were taken at 4 hpi. D: Quantification of ROS biosensor activation in dram1+/+ and dram1-/- at 4hpi. Numbers of phagocytes showing ROS biosensor activation (GFP and mCherry bacterial signals) or without ROS biosensor activation ( $m$ Cherry bacterial signal only) were counted from confocal images and the percentages of ROS biosensor-positive over the total were averaged from five embryos per group. Error bars represent the SD. Scale bar $(B-C)=10 \mu \mathrm{m} . * * * * \mathrm{P}<0.0001$. 


\section{Figure 1}

\section{A}

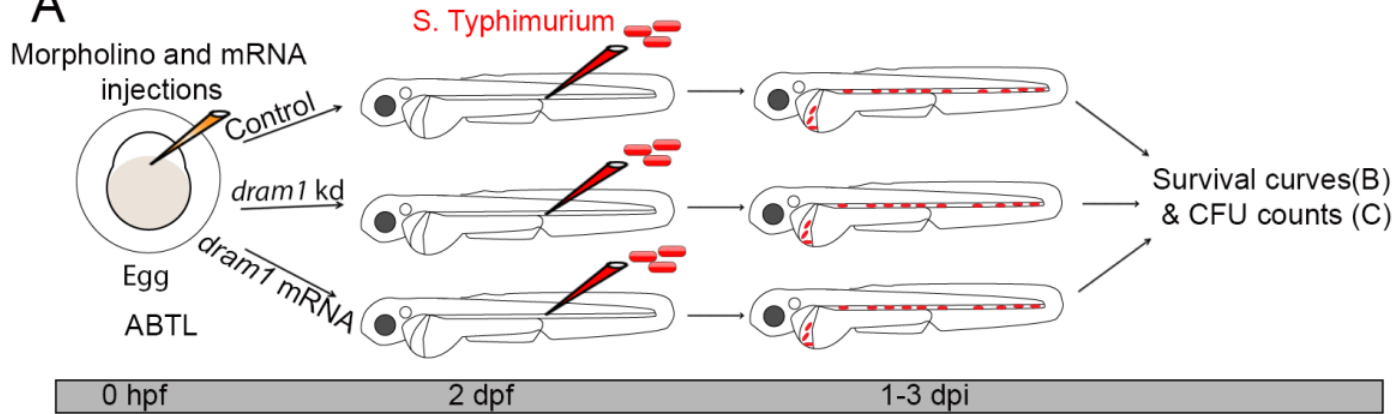
Time line

B

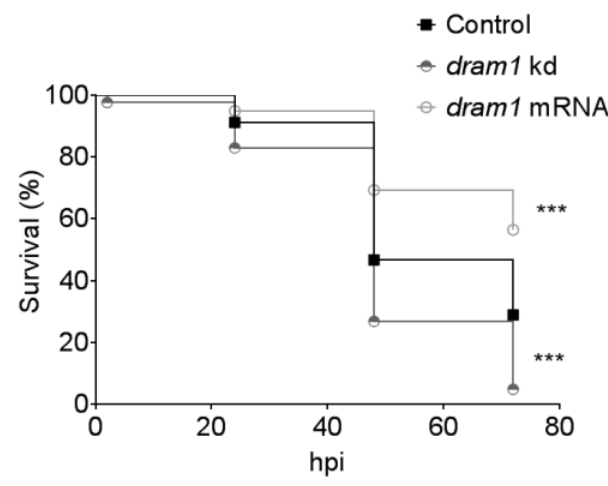

C

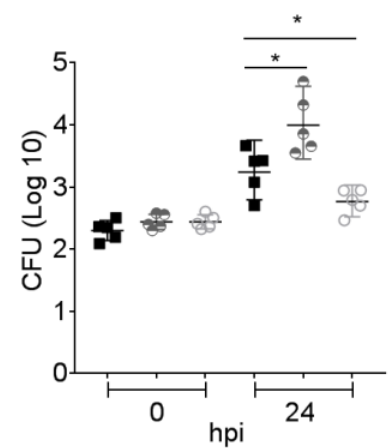




\section{Figure 2}

\section{A}

Morpholino and mRNA

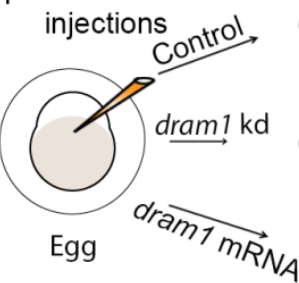

$\operatorname{Tg}(\mathrm{CMV}$ :GFP-map1Lc3b)

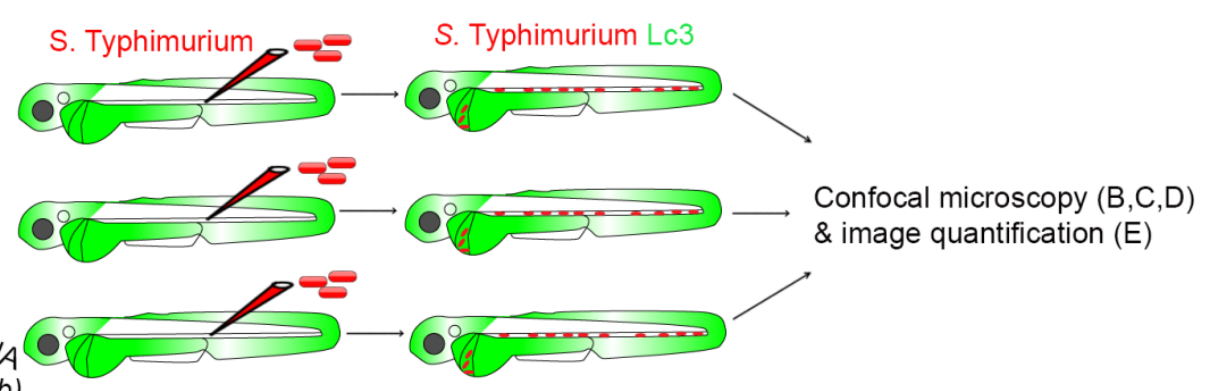

$0 \mathrm{hpf}$

Time line
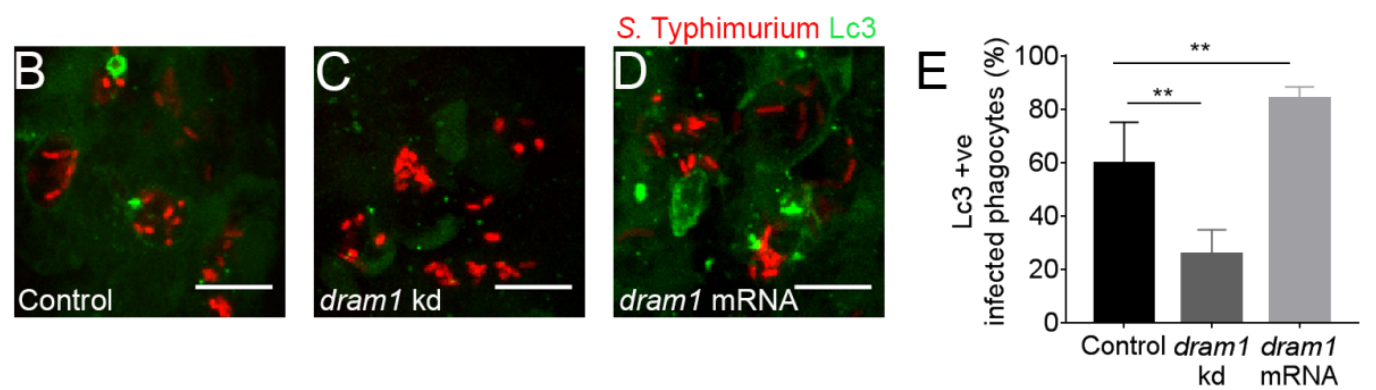


\section{Figure 3}

\section{A}
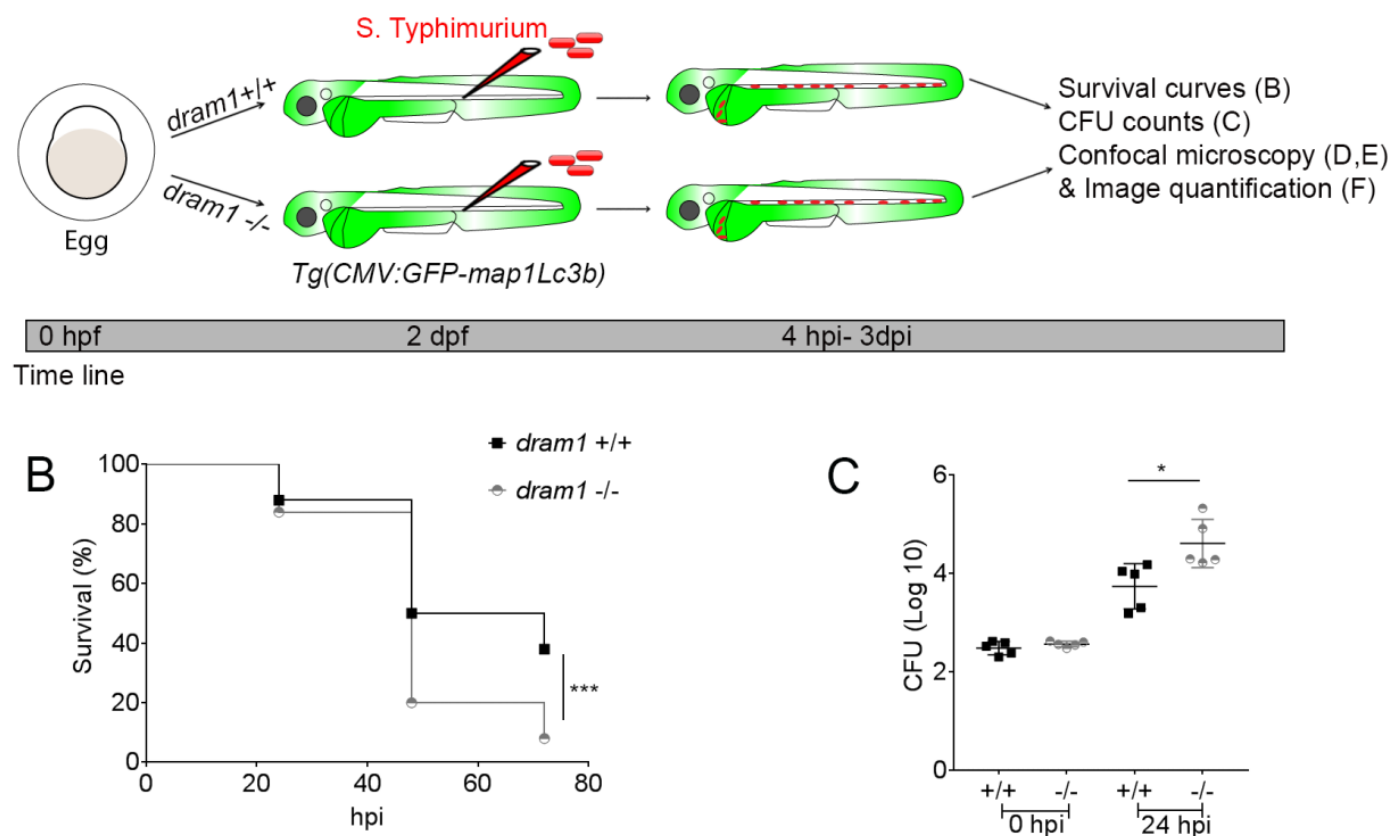

4 hpi- 3dpi
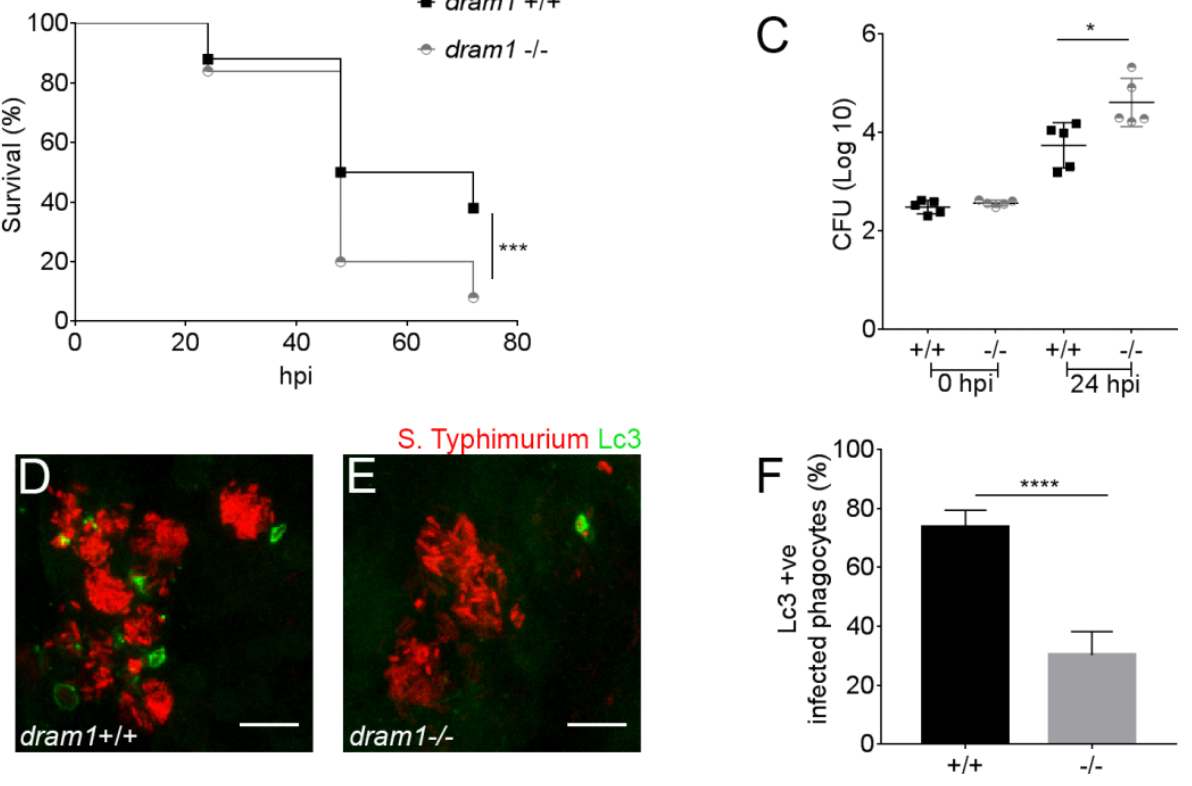


\section{Figure 4}

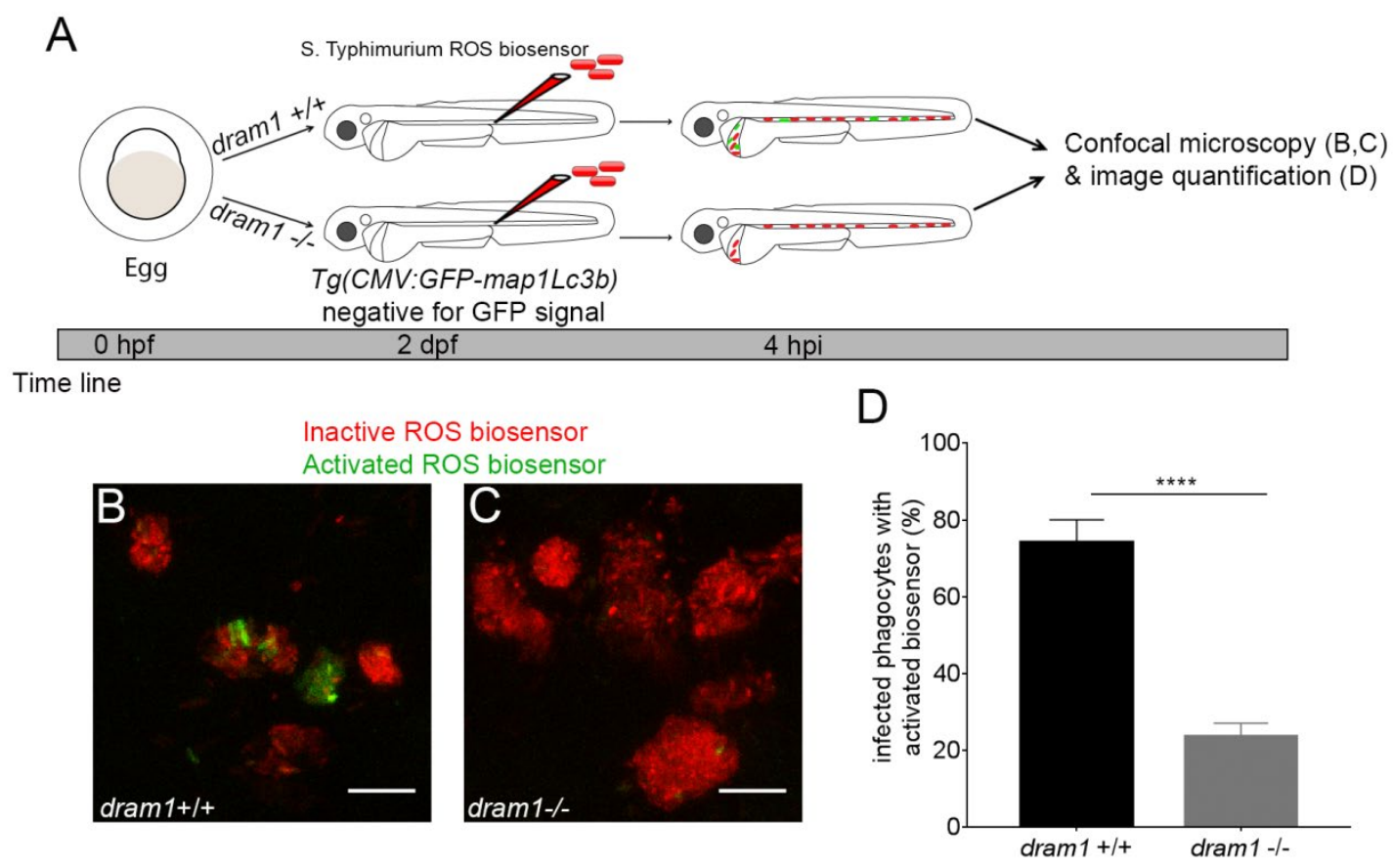

